East Asian Mathematical Journal

Vol. 30 (2014), No. 1, pp. 031-050

http://dx.doi.org/10.7858/eamj.2014.004

\title{
LIMSUP RESULTS AND LIL FOR PARTIAL SUMS OF RANDOM SEQUENCES
}

\author{
Chang-Ho Han, Hee-Jin Moon, and Yong-Kab ChOi*
}

\begin{abstract}
In this paper we establish limsup results and a generalized uniform law of the iterated logarithm (LIL) for the increments of partial sums of strictly stationary and linearly positive quadrant dependent (LPQD) or linearly negative quadrant dependent (LNQD) random sequences.
\end{abstract}

\section{Introduction and Results}

Let $\left\{X_{k} ; k=1,2, \cdots\right\}$ be a sequence of independent identically distributed (i.i.d.) random variables, and let $S_{x}=\sum_{k=1}^{[x]} X_{k}$ and $S_{0}=0$, where $[x]$ denotes the integer part of $x \geq 1$. For a sequence $\left\{a_{n} ; n=1,2, \cdots\right\}$ with $1 \leq a_{n} \leq n$, Csörgő and Révész [6] obtained the following strong limit law

$$
\lim _{n \rightarrow \infty} \max _{0 \leq i \leq n-a_{n}} \max _{1 \leq j \leq a_{n}} \frac{S_{i+j}-S_{i}}{\sqrt{2 a_{n}\left(\log \left(n / a_{n}\right)+\log \log n\right)}}=1 \quad \text { a.s. }
$$

under some conditions of $\left\{X_{k}\right\}$ and $\left\{a_{n}\right\}$. The result (1.1) generalizes the wellknown "new law of large numbers" by Erdös and Rényi [11]. For further various results on this limit law (1.1), we refer to [9, 10, 15]. Csörgö and Révész [7, 8] also considered continuous random processes, for example, Wiener process in place of the partial sum of i.i.d. random variables in (1.1).

On the other hand, Lin $[18,19]$ established several results related to (1.1) for a sequence of independent or mixing dependent random variables. Theoretically and practically, strong dependent sequences are important and interesting. Usually one considers the case of Gaussian sequences. Recently, Choi et al. [4] investigated almost sure limit theorems for the maximum of partial sums of stationary Gaussian sequences under dependent conditions. Besides, Csörgő et al. [5] and Choi and Csörgö [2] studied path properties of $l^{\infty}$ and $l^{p}$-valued Gaussian random fields, respectively.

Received August 9, 2013; Accepted December 3, 2013.

2000 Mathematics Subject Classification. 60F15, 60G10, 60G17.

Key words and phrases. quadrant dependence, stationary random variable, law of the iterated logarithm.

*Corresponding author. This work was financially supported by NRF-2013R1A1A2010278. 
In the last 50 years, there has been growing interest in concepts of positive or negative dependence for families of random variables. Such concepts are used in deriving inequalities in probability and statistics. In this paper, we are interested in the asymptotic properties for a random sequence under dependence assumptions.

For the aim of the present paper, we need to elaborate upon definitions which will play a basic role in our work. For an integer $d \geq 1$, let $\mathbb{R}^{d}$ and $\mathbb{R}_{+}^{d}$, respectively, be $d$-dimensional and nonnegative $d$-dimensional Euclidean spaces with the coordinatewise partial order $\leq$, where $\mathbb{R}$ is the set of real numbers. A sequence $\left\{X_{k} ; k=1,2, \cdots\right\}$ of real-valued random variables on the probability space $(\Omega, \mathfrak{F}, P)$ is said to be centered if $E\left(X_{k}\right)=0$.

Esary et al. [12] and Joag-Dev and Proschan [14] introduced definitions of positive association and negative association, respectively: Let $\mathcal{C}$ be a set of functions of the form $f: \mathbb{R}^{n} \rightarrow \mathbb{R}(n \geq 1)$ which are coordinatewise nondecreasing. A finite family $\left\{X_{1}, \cdots, X_{n}\right\}$ of random variables is said to be positively associated (PA, for short) if, for any $f, g \in \mathcal{C}$ and any subsets $A$ and $B$ of $\{1,2, \cdots, n\}$,

$$
\operatorname{Cov}\left(f\left(X_{i} ; i \in A\right), g\left(X_{j} ; j \in B\right)\right) \geq 0,
$$

while $\left\{X_{1}, \cdots, X_{n}\right\}$ is said to be negatively associated (NA, for short) if, for any $f, g \in \mathcal{C}$ and any disjoint subsets $A$ and $B$ of $\{1,2, \cdots, n\}$,

$$
\operatorname{Cov}\left(f\left(X_{i} ; i \in A\right), g\left(X_{j} ; j \in B\right)\right) \leq 0 .
$$

An infinite family is PA (resp. NA) if every finite subfamily is PA (resp. NA).

Newman [23] introduced and discussed the following another concepts of positive or negative dependence. A sequence $\left\{X_{k} ; k=1,2, \cdots\right\}$ of random variables is said to be linearly positive quadrant dependent (LPQD, for short) if, for any positive numbers $\lambda_{i}$ and any disjoint finite subsets $A, B$ of $\mathbb{Z}_{+}$, the following inequality

$$
P\left\{\sum_{i \in A} \lambda_{i} X_{i} \geq x, \sum_{j \in B} \lambda_{j} X_{j} \geq y\right\} \geq P\left\{\sum_{i \in A} \lambda_{i} X_{i} \geq x\right\} P\left\{\sum_{j \in B} \lambda_{j} X_{j} \geq y\right\}
$$

holds for all $x, y \in \mathbb{R}$, which is equivalent to the following inequality (Lehmann [16], pp. 1137-1138)

$$
P\left\{\sum_{i \in A} \lambda_{i} X_{i} \leq x, \sum_{j \in B} \lambda_{j} X_{j} \leq y\right\} \geq P\left\{\sum_{i \in A} \lambda_{i} X_{i} \leq x\right\} P\left\{\sum_{j \in B} \lambda_{j} X_{j} \leq y\right\},
$$

while $\left\{X_{k} ; k=1,2, \cdots\right\}$ is said to be linearly negative quadrant dependent (LNQD, for short) if the inequalities in (1.2) and (1.3) are reversed. In general, two random variables $X$ and $Y$ have been called positively (resp. negatively) quadrant dependent (PQD) (resp. NQD) by Lehmann [16], if $P(X \geq x, Y \geq$ $y) \geq($ resp. $\leq) P(X \geq x) P(Y \geq y)$ for all $x, y \in \mathbb{R}$. 
From the definitions, it is obvious that PA or NA implies LPQD or LNQD (cf. $[12,14]$ ), respectively, but the converse is not true (see e.g. Joag-Dev [13], pp. 1038-1039). The positive or negative dependence plays an important role in a wide variety of areas, including statistical mechanics, quantum field theory, percolation models, multinomial distribution, permutation distribution, reliability theory, mathematical physics and multivariate statistical analysis.

Since LPQD and LNQD are strictly weaker than PA and NA, respectively, studying the limit theorems for LPQD and LNQD random sequences is of interest. The following is not necessarily an exhaustive list of papers for LPQD and LNQD random variables: [1], [23], [24], [26], [28].

Recently, Li and Wang [17] obtained the following law of the iterated logarithm (LIL) for an LPQD random sequence (cf. Theorem A below). Let $\left\{X_{k} ; k=1,2, \cdots\right\}$ be a centered strictly stationary LPQD random sequence with $E X_{1}^{2}>0$, which satisfies conditions

(i) $E\left|X_{1}\right|^{p}<\infty$ for $p>2$,

(ii) $\sum_{j \geq k+1} \operatorname{Cov}\left(X_{1}, X_{j}\right)=O\left(k^{-\lambda}\right)$ for some $\lambda>2$ and each $k \geq 1$,

(iii) $\sigma^{2}:=E X_{1}^{2}+2 \sum_{j=2}^{\infty} \operatorname{Cov}\left(X_{1}, X_{j}\right)<\infty$

where $u_{k}=O\left(v_{k}\right)$ denotes $\lim \sup _{k \rightarrow \infty} u_{k} / v_{k}<\infty$.

Theorem A (LIL). Let $\left\{X_{k} ; k=1,2, \cdots\right\}$ be a centered strictly stationary $L P Q D$ random sequence with $E X_{1}^{2}>0$, which satisfies conditions (i)-(iii) above. Then

$$
\limsup _{n \rightarrow \infty} \frac{S_{n}}{\sqrt{2 \sigma^{2} n \log \log n}}=1 \quad \text { a.s. }
$$

In this paper, we establish some limsup results and a generalized uniform law of the iterated logarithm for the increments of partial sums of a centered strictly stationary LPQD (or LNQD) sequence of random variables, which extend and generalize the inspiring result Theorem $\mathrm{A}$.

Throughout the paper, let $\left\{X_{k} ; k=1,2, \cdots\right\}$ be a centered strictly stationary LPQD (or LNQD) sequence of random variables with $E\left|X_{1}\right|^{2+\delta}<\infty$ for some $\delta \in(0,1]$. Define a partial sum of the sequence $\left\{X_{k}\right\}$ by $S_{x}=\sum_{k=1}^{[x]} X_{k}$ and $S_{0}=0$, where $[x]$ denotes the integer part of $x \geq 1$. Set

$$
\sigma(x):=\sqrt{E\left(S_{i+x}-S_{i}\right)^{2}}>0, \quad x>0,
$$

for all $i \geq 0$, by stationarity. Assume that $\sigma(x)$ is a nondecreasing and regularly varying function with exponent $\alpha>0$ at $\infty$.

A positive function $R(x)$ of $x>0$ is said to be regularly varying with exponent $\alpha>0$ at $b \geq 0$ if $\lim _{x \rightarrow b}\{R(t x) / R(x)\}=t^{\alpha}$ for $t>0$. Recall that a function $L(x)$ of $x>0$ is said to be slowly varying at $b \geq 0$ if $\lim _{x \rightarrow b}\{L(t x) / L(x)\}=$ 
1 for $t>0$. Thus the regularly varying function $\sigma(x)$ can be written to $\sigma(x)=x^{\alpha} L(x)$.

Under the condition (iii) above, Yang and Wang [8] proved

$$
\lim _{n \rightarrow \infty} \frac{\sigma^{2}(n)}{n}=\sigma^{2}
$$

By this relation, we ensure that $\sigma(n$ is approximately equal to $\sigma \sqrt{n}$ for $n$ large enough, and that $\sigma(\cdot)$ is regarded as a regularly varying function with exponent $\alpha=1 / 2$ at $\infty$. Furthermore, (1.4) can be written as

$$
\limsup _{n \rightarrow \infty} \frac{S_{n}}{\sigma(n) \sqrt{2 \log \log n}}=1 \quad \text { a.s. }
$$

under conditions (i)-(iii).

Suppose that $\left\{a_{n} ; n \geq 1\right\}$ and $\left\{b_{n} ; n \geq 1\right\}$ are positive nondecreasing sequences such that $a_{n} \leq b_{n}$ and $b_{n} \rightarrow \infty$ as $n \rightarrow \infty$. Denote

$$
\beta(n)=\sqrt{2\left\{\log \left(b_{n} / a_{n}\right)+\log \log b_{n}\right\}},
$$

where $\log x:=\log (\max \{x, e\})$. The main results are as follows.

Theorem 1.1. Let $\left\{X_{k} ; k=1,2, \cdots\right\}$ be a centered strictly stationary LPQD (LNQD) random sequence with $E\left|X_{1}\right|^{2+\delta}<\infty$ for some $\delta \in(0,1]$, which satisfies conditions

(i) $\sum_{j \geq k+1}\left|\operatorname{Cov}\left(X_{1}, X_{j}\right)\right|=O\left(k^{-\lambda}\right)$ for some $\lambda>2$ and each $k \geq 1$,

(ii) $\inf _{x \geq 1} \sigma^{2}(x) / x>0$.

Then we have

$$
\begin{aligned}
\limsup _{n \rightarrow \infty} \sup _{0 \leq i \leq b_{n}} & \sup _{1 \leq j \leq b_{n}} \frac{\left|S_{i+j}-S_{i}\right|}{\sigma\left(b_{n}\right) \sqrt{2 \log \log b_{n}}} \\
& =\limsup _{n \rightarrow \infty} \frac{S_{b_{n}}}{\sigma\left(b_{n}\right) \sqrt{2 \log \log b_{n}}}=1 \quad \text { a.s. }
\end{aligned}
$$

The first result in (1.6) implies a generalized uniform law of the iterated logarithm for LPQD or LNQD random sequences, but the second one in (1.6) is a standard form of the ordinary LIL for LPQD or LNQD random sequences which is an extension of (1.4) or (1.5) with $b_{n}=n$. Since $\beta(n) \geq \sqrt{2 \log \log b_{n}}$, it is natural from (1.6) that

$$
\limsup _{n \rightarrow \infty} \sup _{0 \leq i \leq b_{n}} \sup _{1 \leq j \leq b_{n}} \frac{\left|S_{i+j}-S_{i}\right|}{\sigma\left(b_{n}\right) \beta(n)} \leq 1 \quad \text { a.s. }
$$

but it is impossible by the second equality in (1.6) that the following inequality holds:

$$
\limsup _{n \rightarrow \infty} \frac{S_{b_{n}}}{\sigma\left(b_{n}\right) \beta(n)} \geq 1 \quad \text { a.s. }
$$


under the conditions in Theorem 1.1. Hence it is necessary to add the following conditions (iii)-(iv) to Theorem 1.1 as in Theorem 1.2 below in order to obtain (1.8).

Theorem 1.2. Let $\left\{X_{k} ; k=1,2, \cdots\right\}$ be a centered strictly stationary LPQD $(L N Q D)$ random sequence with $E\left|X_{1}\right|^{2+\delta}<\infty$ for some $\delta \in(0,1]$, which satisfies conditions (i)-(ii) in Theorem 1.1. Suppose that sequences $\left\{a_{n} ; n \geq 1\right\}$ and $\left\{b_{n} ; n \geq 1\right\}$ satisfy additional conditions

(iii) $b_{n} / a_{n}(>1)$ is increasing,

(iv) there exists $c_{0}>1$ such that $b_{n} \leq c_{0} b_{n-1}$ for $n \geq 2$.

Then we have

$$
\limsup _{n \rightarrow \infty} \sup _{0 \leq i \leq b_{n}} \sup _{1 \leq j \leq b_{n}} \frac{\left|S_{i+j}-S_{i}\right|}{\sigma\left(b_{n}\right) \beta(n)}=\limsup _{n \rightarrow \infty} \frac{S_{b_{n}}}{\sigma\left(b_{n}\right) \beta(n)}=1 \quad \text { a.s. }
$$

Theorems 1.1 and 1.2 for LPQD or LNQD random sequences generalize the superior limit results in [3], [4], [6], [21] and [22].

\section{Proofs}

In this section, let $c$ denote a positive constant which may take different values whenever it appears in different lines. We need the following properties.

$\left(P_{1}\right)$ Two random variables $X$ and $Y$ are PQD (resp. NQD) if and only if $\operatorname{Cov}(f(X), g(Y)) \geq($ resp. $\leq) 0$ for all real-valued nondecreasing functions $f$ and $g$ (such that $f(X)$ and $g(Y)$ have finite variances) (see Lehmann [16]);

$\left(P_{2}\right)$ (Hoeffding equality): For any absolutely continuous functions $f$ and $g$ on the real line and for any random variables $X$ and $Y$ satisfying $E f^{2}(X)+$ $E g^{2}(Y)<\infty$, we have

$$
\begin{aligned}
\operatorname{Cov}( & f(X), g(Y)) \\
= & \int_{-\infty}^{\infty} \int_{-\infty}^{\infty} f^{\prime}(x) g^{\prime}(y)\{P(X \geq x, Y \geq y)-P(X \geq x) P(Y \geq y)\} d x d y .
\end{aligned}
$$

The following propositions are essential to prove our main theorems. Note that the condition (i) in Theorem 1.1 for strictly stationary LPQD (LNQD) random sequences implies conditions (C2) and (I) in [17] and [26], respectively. Thus Lemma 2 in [17] and Corollary 2.1 in [26] are easily changed to the following Berry-Esseen type theorem for strictly stationary LPQD (LNQD) random sequences in normal approximation under the assumptions of Theorem 1.1 .

Proposition 2.1. (Berry-Esseen type theorem). Let $\left\{X_{k} ; k=1,2, \cdots\right\}$ be a centered strictly stationary LPQD (LNQD) random sequence with $E\left|X_{1}\right|^{2+\delta}<$ 
$\infty$ for some $\delta \in(0,1]$, which satisfies conditions (i)-(ii) in Theorem 1.1. Then

$$
\sup _{z}\left|P\left\{\frac{S_{b_{n}}}{\sigma\left(b_{n}\right)} \leq z\right\}-\Phi(z)\right|=O\left(b_{n}^{-1 / 5}\right), \quad n \rightarrow \infty
$$

where $\Phi(\cdot)$ is a standard normal distribution function and $b_{n} \rightarrow \infty$ as $n \rightarrow \infty$.

Denote $b_{k}=b_{n_{k}}$ for a nonnegative increasing sequence $\left\{n_{k}\right\}_{k=1}^{\infty}$. Using Proposition 2.1 above, the following proposition is immediate from the proof of Lemma 9 in Petrov [25, p. 311].

Proposition 2.2. Let $\left\{X_{k}\right\}$ be as in Proposition 2.1. Assume that $g(x)$ is a positive nondecreasing function of $x>0$ and $\left\{b_{k} ; k \geq 1\right\}$ is a positive nondecreasing sequence such that $\sum_{k=1}^{\infty} b_{k}^{-1 / 5}<\infty$. Then the following statements are equivalent:
(A) $\sum_{k=1}^{\infty} P\left\{\frac{S_{b_{k}}}{\sigma\left(b_{k}\right)}>g\left(b_{k}\right)\right\}<\infty$,
(B) $\sum_{k=1}^{\infty} P\left\{\frac{\left|S_{b_{k}}\right|}{\sigma\left(b_{k}\right)}>g\left(b_{k}\right)\right\}<\infty$,
(C) $\sum_{k=1}^{\infty} \frac{1}{g\left(b_{k}\right)} \exp \left(-\frac{1}{2} g^{2}\left(b_{k}\right)\right)<\infty$.

The following proposition on the large deviation probability will be proved in Section 3.

Proposition 2.3. Let $\left\{X_{k} ; k=1,2, \cdots\right\}$ be a centered strictly stationary random sequence. Then, for any $\varepsilon>0$ there exists a positive constant $c_{\varepsilon}$ such that, for $u>1$,

$$
\begin{aligned}
& P\left\{\sup _{0 \leq i \leq b_{n}} \sup _{1 \leq j \leq b_{n}} \frac{\left|S_{i+j}-S_{i}\right|}{\sigma\left(b_{n}\right)} \geq u\right\} \\
& \leq c_{\varepsilon}\left(P\left\{\frac{\left|S_{b_{n}}\right|}{\sigma\left(b_{n}\right)} \geq \frac{u}{1+\varepsilon}\right\}+\sum_{n=1}^{\infty} 2^{2^{n+1}} P\left\{\frac{\left|S_{b_{n}}\right|}{\sigma\left(b_{n}\right)} \geq \frac{u}{1+\varepsilon} \sqrt{1+2 \log 3} \cdot 2^{n / 2}\right\}\right) .
\end{aligned}
$$

Proof of Theorem 1.1. Let us first prove

$$
\limsup _{n \rightarrow \infty} \sup _{0 \leq i \leq b_{n}} \sup _{1 \leq j \leq b_{n}} \frac{\left|S_{i+j}-S_{i}\right|}{\sigma\left(b_{n}\right) \sqrt{2 \log \log b_{n}}} \leq 1 \quad \text { a.s. }
$$

For any $\theta>1$, set $A_{k}=\left\{n ; \theta^{k-1} \leq b_{n} \leq \theta^{k}\right\}, k \geq 1$. Note that $\sqrt{2 \log \log \theta^{k-1}} \geq$ $\theta^{-1} \sqrt{2 \log \log \theta^{k}}$ since $(\log u) / u$ is decreasing for any $u>e^{e}$. By the regularity 
of $\sigma(\cdot)$, we get $\sigma\left(b_{n}\right) / \sigma\left(\theta^{k}\right) \geq \theta^{-2 \alpha}$ as $k \rightarrow \infty$ and hence

$$
\begin{aligned}
& \limsup _{n \rightarrow \infty} \sup _{0 \leq i \leq b_{n}} \sup _{1 \leq j \leq b_{n}} \frac{\left|S_{i+j}-S_{i}\right|}{\sigma\left(b_{n}\right) \sqrt{2 \log \log b_{n}}} \\
& \leq \limsup _{k \rightarrow \infty} \sup _{n \in \mathbb{A}_{k}} \sup _{0 \leq i \leq b_{n}} \sup _{1 \leq j \leq b_{n}} \frac{\left|S_{i+j}-S_{i}\right|}{\sigma\left(b_{n}\right) \sqrt{2 \log \log \theta^{k-1}}} \\
& \leq \theta^{2 \alpha+1} \limsup _{k \rightarrow \infty} \sup _{0 \leq i \leq \theta^{k}} \sup _{1 \leq j \leq \theta^{k}} \frac{\left|S_{i+j}-S_{i}\right|}{\sigma\left(\theta^{k}\right) \sqrt{2 \log \log \theta^{k}}} .
\end{aligned}
$$

Let us apply Proposition 2.3 with $b_{k}=\theta^{k}$, where $b_{k}:=b_{n_{k}}$ for an increasing subsequence $\left\{n_{k}\right\}_{k=1}^{\infty}$ of $\{n ; n \geq 1\}$. Then, for any $\varepsilon>0$, there exists a constant $c_{\varepsilon}>0$ such that

$$
\begin{aligned}
& P\left\{\sup _{0 \leq i \leq \theta^{k}} \sup _{1 \leq j \leq \theta^{k}} \frac{\left|S_{i+j}-S_{i}\right|}{\sigma\left(\theta^{k}\right) \sqrt{2 \log \log \theta^{k}}}>1+2 \varepsilon\right\} \\
& \leq c_{\varepsilon}\left(P\left\{\frac{\left|S_{\theta^{k}}\right|}{\sigma\left(\theta^{k}\right)}>\frac{1+2 \varepsilon}{1+\varepsilon} \sqrt{2 \log \log \theta^{k}}\right\}\right. \\
& \left.+\sum_{n=1}^{\infty} 2^{2^{n+1}} P\left\{\frac{\left|S_{\theta^{k}}\right|}{\sigma\left(\theta^{k}\right)}>\frac{(1+2 \varepsilon) \sqrt{2 \log \log \theta^{k}}}{1+\varepsilon} \sqrt{1+2 \log 3} \cdot 2^{n / 2}\right\}\right)
\end{aligned}
$$

for $k$ large enough. Now, let us apply Proposition 2.2 with $b_{k}=\theta^{k}$ and $g\left(\theta^{k}\right)=$ $g_{1}\left(\theta^{k}\right)\left(\right.$ or $\left.g_{2}\left(\theta^{k}\right)\right)$, where

$$
\begin{aligned}
g_{1}\left(\theta^{k}\right) & :=\frac{(1+2 \varepsilon) \sqrt{2 \log \log \theta^{k}}}{1+\varepsilon}, \\
g_{2}\left(\theta^{k}\right) & :=\frac{(1+2 \varepsilon) \sqrt{2 \log \log \theta^{k}}}{1+\varepsilon} \sqrt{1+2 \log 3} \cdot 2^{n / 2} .
\end{aligned}
$$

Considering the first term of the right hand side of (2.3) and (C) of Proposition 2.2 , we have

$$
\begin{aligned}
\sum_{k=1}^{\infty} \frac{1}{g_{1}\left(\theta^{k}\right)} \exp \left(-\frac{1}{2} g_{1}^{2}\left(\theta^{k}\right)\right) & \leq c \sum_{k=1}^{\infty} \exp \left(-\frac{1}{2}\left(\frac{1+2 \varepsilon}{1+\varepsilon}\right)^{2} 2 \log \log \theta^{k}\right) \\
& \leq c \sum_{k=1}^{\infty}\left(\log \theta^{k}\right)^{-1-\varepsilon^{\prime}}<\infty
\end{aligned}
$$

where $\varepsilon^{\prime}=\varepsilon /(1+\varepsilon)$, and hence Proposition 2.2 yields

$$
\sum_{k=1}^{\infty} P\left\{\frac{\left|S_{\theta^{k}}\right|}{\sigma\left(\theta^{k}\right)}>g_{1}\left(\theta^{k}\right)\right\}<\infty .
$$

Next, consider the second term of the right hand side of (2.3). By the equivalence relation of (B) and (C) in Proposition 2.2 with $g\left(\theta^{k}\right)=g_{2}\left(\theta^{k}\right)$, it is 
obvious that

$$
\begin{aligned}
\sum_{k=1}^{\infty} \frac{1}{g_{2}\left(\theta^{k}\right)} \exp \left(-\frac{1}{2} g_{2}^{2}\left(\theta^{k}\right)\right)<\infty & \\
\Longrightarrow & \sum_{k=1}^{\infty} P\left\{\frac{\left|S_{\theta^{k}}\right|}{\sigma\left(\theta^{k}\right)}>g_{2}\left(\theta^{k}\right)\right\}<\infty
\end{aligned}
$$

By the way, we have

$$
\begin{aligned}
\frac{1}{g_{2}\left(\theta^{k}\right)} & \exp \left(-\frac{1}{2} g_{2}^{2}\left(\theta^{k}\right)\right) \\
& \leq \exp \left(-\frac{1}{2}\left(\frac{1+2 \varepsilon}{1+\varepsilon}\right)^{2}\left(2 \log \log \theta^{k}\right)(1+2 \log 3) 2^{n}\right) \\
& \leq(k \log \theta)^{-\left(1+\varepsilon^{\prime}\right)(1+2 \log 3) 2^{n}} \\
& \leq c k^{-\left(1+\varepsilon^{\prime}\right)(1+2 \log 3) 2^{n}}
\end{aligned}
$$

for some constant $c>0$, and hence the implication of (2.5) gives

$$
P\left\{\frac{\left|S_{\theta^{k}}\right|}{\sigma\left(\theta^{k}\right)}>g_{2}\left(\theta^{k}\right)\right\} \leq c k^{-\left(1+\varepsilon^{\prime}\right)(1+2 \log 3) 2^{n}} .
$$

Therefore, we obtain

$$
\begin{aligned}
\sum_{k=1}^{\infty} & \sum_{n=1}^{\infty} 2^{2^{n+1}} P\left\{\frac{\left|S_{\theta^{k}}\right|}{\sigma\left(\theta^{k}\right)}>g_{2}\left(\theta^{k}\right)\right\} \\
& \leq c \sum_{k=1}^{\infty} \sum_{n=1}^{\infty} 2^{2^{n+1}} k^{-\left(1+\varepsilon^{\prime}\right)(1+2 \log 3) 2^{n}} \\
& =c \sum_{k=1}^{\infty} \sum_{n=1}^{\infty} 2^{2^{n+1}} \cdot 2^{-\left(\log _{2} k\right)\left(1+\varepsilon^{\prime}\right) 2^{n}} \cdot 2^{-\left(\log _{2} k\right)\left(1+\varepsilon^{\prime}\right) 2^{n+1} \log 3} \\
& \leq c \sum_{k=1}^{\infty} \sum_{n=1}^{\infty} 2^{2^{n+1}}\left(1-\left(\log _{2} k\right)\left(1+\varepsilon^{\prime}\right) \log 3\right) \cdot 2^{-\left(\log _{2} k\right) 2^{n}} \\
& \leq c \sum_{k=1}^{\infty} \sum_{n=1}^{\infty} 2^{-2\left(1+\varepsilon^{\prime}\right) \log _{2} k} \cdot 2^{-n} \leq c \sum_{k=1}^{\infty} \sum_{n=1}^{\infty} k^{-2} \cdot 2^{-n}<\infty
\end{aligned}
$$

It follows from (2.3), (2.4) and (2.6) that

$$
\sum_{k=1}^{\infty} P\left\{\sup _{0 \leq i \leq \theta^{k}} \sup _{1 \leq j \leq \theta^{k}} \frac{\left|S_{i+j}-S_{i}\right|}{\sigma\left(\theta^{k}\right) \sqrt{2 \log \log \theta^{k}}}>1+2 \varepsilon\right\}<\infty
$$

and the Borel-Cantelli lemma yields

$$
\limsup _{k \rightarrow \infty} \sup _{0 \leq i \leq \theta^{k}} \sup _{1 \leq j \leq \theta^{k}} \frac{\left|S_{i+j}-S_{i}\right|}{\sigma\left(\theta^{k}\right) \sqrt{2 \log \log \theta^{k}}} \leq 1+2 \varepsilon \quad \text { a.s. }
$$

This and (2.2) together imply (2.1) since $\theta$ and $\varepsilon$ are arbitrary. 
By virtue of (2.1), the proof of (1.6) is completed if we show that

$$
\limsup _{k \rightarrow \infty} \frac{S_{b_{k}}}{\sigma\left(b_{k}\right) \sqrt{2 \log \log b_{k}}}>1-4 \varepsilon \quad \text { a.s. }
$$

for any small $\varepsilon>0$, where $b_{k}:=b_{n_{k}}$ for an increasing subsequence $\left\{n_{k}\right\}_{k=1}^{\infty}$ of $\{n ; n \geq 1\}$. Let

$$
B_{k}=\left\{\frac{S_{b_{k}}-S_{b_{k / 2}}}{\sigma\left(b_{k}-b_{k / 2}\right)}>(1-2 \varepsilon) \sqrt{2 \log \log \left(b_{k}-b_{k / 2}\right)}\right\} .
$$

Note that

$$
Z_{k}:=\frac{S_{b_{k}}-S_{b_{k / 2}}}{\sigma\left(b_{k}-b_{k / 2}\right)}
$$

is a standardized random variable. For $\theta>1$, let $b_{k}=\theta^{k}$. Then $b_{k}-b_{k / 2}$ is approximately equal to $\theta^{k}$ for sufficiently large $k$. To apply Proposition 2.2 with $b_{k}-b_{k / 2}$, we set $g\left(b_{k}-b_{k / 2}\right)=(1-2 \varepsilon) \sqrt{2 \log \log \left(b_{k}-b_{k / 2}\right)}$ in $B_{k}$. Then

$$
\begin{aligned}
& \sum_{k=1}^{\infty} \frac{1}{g\left(b_{k}-b_{k / 2}\right)} \exp \left(-\frac{1}{2} g^{2}\left(b_{k}-b_{k / 2}\right)\right) \\
& \quad \geq c \sum_{k=1}^{\infty} \exp \left(-(1-\varepsilon) \log \log \theta^{k}\right) \geq c \sum_{k=1}^{\infty} k^{-1+\varepsilon}=\infty
\end{aligned}
$$

Hence, Proposition 2.2 implies

$$
\sum_{k=1}^{\infty} P\left(B_{k}\right)=\infty
$$

Next, let

We will show that

$$
B_{k}^{\prime}=\left\{Z_{k}>(1-3 \varepsilon) \sqrt{2 \log \log \left(b_{k}-b_{k / 2}\right)}\right\} .
$$

$$
P\left(B_{k}^{\prime}, \text { i.o. }\right)=1 \text {. }
$$

Choose a differential function $f(x)$ on $\mathbb{R}$ such that $\left|f^{\prime}(x)\right| \leq \kappa$ for some $0<$ $\kappa<\infty$ and

$$
\begin{aligned}
0 & \leq I\left\{x>(1-2 \varepsilon) \sqrt{2 \log \log \left(b_{k}-b_{k / 2}\right)}\right\} \\
& \leq f(x) \leq I\left\{x>(1-3 \varepsilon) \sqrt{2 \log \log \left(b_{k}-b_{k / 2}\right)}\right\} \leq 1,
\end{aligned}
$$

where $I(\cdot)$ is an indicator function. In order to prove $(2.9)$, it is enough to show that

$$
\sum_{k=1}^{\infty} f\left(Z_{k}\right)=\infty \quad \text { a.s. }
$$

From (2.8) and (2.10), we get

$$
\sum_{k=1}^{\infty} E f\left(Z_{k}\right) \geq \sum_{k=1}^{\infty} P\left(B_{k}\right)=\infty
$$


By Markov's inequality, we have

$$
\begin{aligned}
& P\left\{\sum_{k=1}^{\infty} f\left(Z_{k}\right)<\frac{1}{2} \sum_{k=1}^{n} E f\left(Z_{k}\right)\right\} \\
& \quad \leq P\left\{\left|\sum_{k=1}^{n} f\left(Z_{k}\right)-\sum_{k=1}^{n} E f\left(Z_{k}\right)\right|>\frac{1}{2} \sum_{k=1}^{n} E f\left(Z_{k}\right)\right\} \\
& \quad \leq 4 \operatorname{Var}\left(\sum_{k=1}^{n} f\left(Z_{k}\right)\right) /\left(\sum_{k=1}^{n} E f\left(Z_{k}\right)\right)^{2} \\
& \leq \frac{4}{\sum_{k=1}^{n} E f\left(Z_{k}\right)}+\frac{8 \sum_{k=1}^{\infty} \sum_{j=k+1}^{\infty}\left|\operatorname{Cov}\left(f\left(Z_{k}\right), f\left(Z_{j}\right)\right)\right|}{\left(\sum_{k=1}^{n} E f\left(Z_{k}\right)\right)^{2}} .
\end{aligned}
$$

Noting that $Z_{k}$ and $Z_{j}$ are LPQD (resp. LNQD) from the definition of LPQD (resp. LNQD), it follows from $(\mathrm{i}),\left(P_{1}\right),\left(P_{2}\right)$ and the regularity of $\sigma(\cdot)$ that

$$
\begin{aligned}
& \sum_{k=1}^{\infty} \sum_{j=k+1}^{\infty}\left|\operatorname{Cov}\left(f\left(Z_{k}\right), f\left(Z_{j}\right)\right)\right| \\
& \leq \sum_{k=1}^{\infty} \sum_{j=k+1}^{\infty} \int_{-\infty}^{\infty} \int_{-\infty}^{\infty}\left|f^{\prime}(x)\right|\left|f^{\prime}(y)\right| \mid P\left\{Z_{k} \geq x, Z_{j} \geq y\right\} \\
& -P\left\{Z_{k} \geq x\right\} P\left\{Z_{j} \geq y\right\} \mid d x d y \\
& \leq \kappa^{2} \sum_{k=1}^{\infty} \sum_{j=k+1}^{\infty} \mid \int_{-\infty}^{\infty} \int_{-\infty}^{\infty}\left(P\left\{Z_{k} \geq x, Z_{j} \geq y\right\}\right. \\
& \left.-P\left\{Z_{k} \geq x\right\} P\left\{Z_{j} \geq y\right\}\right) d x d y \\
& =\kappa^{2} \sum_{k=1}^{\infty} \sum_{j=k+1}^{\infty}\left|\operatorname{Cov}\left(Z_{k}, Z_{j}\right)\right| \\
& \leq c \sum_{k=1}^{\infty} \frac{1}{\sigma^{2}\left(b_{k}-b_{k / 2}\right)} \sum_{j=k+1}^{\infty}\left|\operatorname{Cov}\left(S_{b_{k}}-S_{b_{k / 2}}, S_{b_{j}}-S_{b_{j / 2}}\right)\right| \\
& \leq c \sum_{k=1}^{\infty} \frac{b_{k}-b_{k / 2}}{\sigma^{2}\left(b_{k}-b_{k / 2}\right)} \sum_{j \geq k+1}\left|\operatorname{Cov}\left(X_{1}, S_{b_{j}}-S_{b_{j / 2}}\right)\right| \\
& \leq c \sum_{k=1}^{\infty} \theta^{k(1-2 \alpha)} b_{k+1} \sum_{b_{j} \geq b_{k}+1}\left|\operatorname{Cov}\left(X_{1}, X_{b_{j}}\right)\right| \\
& \leq c \sum_{k=1}^{\infty} \theta^{k(1-2 \alpha)} \theta^{k+1} b_{k}^{-\lambda} \leq c \sum_{k=1}^{\infty} \theta^{k(1-2 \alpha)} \theta^{k} \theta^{-\lambda k} \\
& =c \sum_{k=1}^{\infty} \theta^{-(\lambda-2+2 \alpha) k}<\infty
\end{aligned}
$$


for $\alpha>0$ and $\lambda>2$. Combining (2.12)-(2.14) and letting $n \rightarrow \infty$ yields

$$
P\left\{\sum_{k=1}^{\infty} f\left(Z_{k}\right)<\infty\right\}=0 .
$$

This proves (2.11) and consequently (2.9). Let

$$
C_{k}=\left\{\frac{S_{b_{k / 2}}}{\sigma\left(b_{k / 2}\right)} \geq-2 \sqrt{2 \log \log b_{k / 2}}\right\} .
$$

It follows from $(2.1)$ and $(2.9)$ that $P\left(B_{k}^{\prime} \cap C_{k}\right.$, i.o. $)=1$. It is easy to see that

$$
\begin{aligned}
& P\left\{\frac{S_{b_{k}}}{\sigma\left(b_{k}\right)}>(1-4 \varepsilon) \sqrt{2 \log \log b_{k}}, \text { i.o. }\right\} \\
& \quad \geq P\left\{\frac{S_{b_{k}}}{\sigma\left(b_{k}\right)}>(1-3 \varepsilon) \sqrt{2 \log \log \left(b_{k}-b_{k / 2}\right)}-2 \sqrt{2 \log \log b_{k / 2}}, \text { i.o. }\right\} \\
& \quad \geq P\left\{B_{k}^{\prime} \cap C_{k}, \text { i.o. }\right\}=1
\end{aligned}
$$

for $k$ large enough. This implies (2.7) and completes the proof of Theorem 1.1.

To prove Theorem 1.2, we need the following lemma which is a well-known version of the second Borel-Cantelli lemma.

Lemma 2.4. Let $\left\{\mathbb{A}_{k} ; k \geq 1\right\}$ be any sequence of events in a probability space $(\Omega, \mathfrak{F}, P)$. If

(a) $\sum_{k=1}^{\infty} P\left(\mathbb{A}_{k}\right)=\infty$ and

(b) $\liminf \operatorname{in}_{n \rightarrow \infty} \sum_{1 \leq j<k \leq n} \frac{P\left(\mathbb{A}_{j} \cap \mathbb{A}_{k}\right)-P\left(\mathbb{A}_{j}\right) P\left(\mathbb{A}_{k}\right)}{\left(\sum_{j=1}^{n} P\left(\mathbb{A}_{j}\right)\right)^{2}} \leq 0$,

then $P\left(\lim \sup _{k \rightarrow \infty} \mathbb{A}_{k}\right)=1$.

Proof of Theorem 1.2. Let $\left\{n_{k}\right\}_{k=1}^{\infty}$ be a nonnegative increasing sequence such that $n_{0} \geq 0, \lim _{k \rightarrow \infty} n_{k}=\infty$ and the $(k-1)$ st point $n_{k-1}$ is placed by the relation

$$
b_{n_{k}}-a_{n_{k}}=b_{n_{k-1}}
$$

with $n_{k}(k=1,2, \cdots)$ defined by induction, where $\lim _{k \rightarrow \infty} b_{n_{k}}=\infty$ by assumption. This can be done by the fact that $b_{n_{k}}-a_{n_{k}}$ is increasing since $b_{n_{k}} / a_{n_{k}}(>1)$ is increasing by (iii). For convenience, put $a_{k}=a_{n_{k}}$ and $b_{k}=b_{n_{k}}$, and set

$$
U_{k}=\frac{S_{b_{k}}}{\sigma\left(b_{k}\right)}
$$

By virtue of (1.7), the proof of (1.9) is completed if we show that

$$
\limsup _{k \rightarrow \infty} \frac{U_{k}}{\beta\left(n_{k}\right)}>\sqrt{1-4 \varepsilon} \quad \text { a.s. }
$$


for any small $\varepsilon>0$, where $\beta\left(n_{k}\right):=\sqrt{2 \log \left(\left(b_{k} / a_{k}\right) \log b_{k}\right)}$. Set

$$
\mathbb{A}_{k}=\left\{U_{k}>\sqrt{1-4 \varepsilon} \beta\left(n_{k}\right)\right\} .
$$

Let us first prove that $\sum_{k=1}^{\infty} P\left(\mathbb{A}_{k}\right)=\infty$ in Lemma 2.1. Put

$$
\mathbb{B}_{k}=\left\{U_{k}>\sqrt{1-2 \varepsilon} \beta\left(n_{k}\right)\right\} .
$$

If $\mathcal{N}$ is a standard normal random variable, then it is well known that, for all large $x>0$,

$$
\frac{1}{\sqrt{2 \pi} x^{2}} e^{-x^{2} / 2} \leq P\{\mathcal{N}>x\} \leq \frac{1}{\sqrt{2 \pi} x} e^{-x^{2} / 2}
$$

Thus it follows from Proposition 2.1 and the stationarity of $\left\{X_{k}\right\}$ that, for all large $k$,

$$
\begin{aligned}
P\left(\mathbb{B}_{k}\right) & =\left(1-P\left\{\frac{S_{b_{k}}}{\sigma\left(b_{k}\right)} \leq \sqrt{1-2 \varepsilon} \beta\left(n_{k}\right)\right\}-1+P\left\{\mathcal{N} \leq \sqrt{1-2 \varepsilon} \beta\left(n_{k}\right)\right\}\right) \\
& \geq-c_{1} b_{k}^{-1 / 5}+P\left\{\mathcal{N}>\sqrt{1-2 \varepsilon} \beta\left(n_{k}\right)\right\} \\
& \geq-c_{1} b_{k}^{-1 / 5}+\frac{1}{\sqrt{2 \pi}(1-2 \varepsilon) \beta^{2}\left(n_{k}\right)} \exp \left(-\frac{1-2 \varepsilon}{2} \beta^{2}\left(n_{k}\right)\right) \\
& \geq-c_{1} b_{k}^{-1 / 5}+c\left(\frac{a_{k}}{b_{k} \log b_{k}}\right)^{1-\varepsilon} \\
& \geq-c_{1} \frac{1}{\left(\log b_{k}\right)^{1-\varepsilon}}+c\left(\frac{a_{k}}{b_{k} \log b_{k}}\right)^{1-\varepsilon} \\
& \geq c \frac{1}{\left(\log b_{k}\right)^{1-\varepsilon}}\left(\varepsilon \frac{a_{k}}{b_{k}}\right)
\end{aligned}
$$

where $c$ and $c_{1}$ are positive constants, and further

$$
\sum_{k=k_{0}}^{m} P\left(\mathbb{B}_{k}\right) \geq \varepsilon \frac{1}{\left(\log b_{m}\right)^{1-\varepsilon}} \sum_{k=k_{0}}^{m} \frac{a_{k}}{b_{k}}
$$

for some $k_{0} \geq 1$ with $k_{0} \leq k \leq m$. Also, by (iv), there exist constants $c_{0}, c_{2}>1$ such that $c_{0} b_{k-1} \geq b_{k}-a_{k}$ and

$$
\begin{aligned}
\log b_{m} & \leq c_{2} \sum_{k=k_{0}}^{m} \log \frac{b_{k}}{b_{k-1}} \leq c_{2} \sum_{k=k_{0}}^{m} \log \left(c_{0}+\frac{a_{k}}{b_{k-1}}\right) \\
& \leq c_{2} \sum_{k=k_{0}}^{m} \log \left(c_{0}+\frac{c_{3} a_{k-1}}{b_{k-1}}\right)
\end{aligned}
$$


for sufficiently large $c_{3}>1$. The last inequality of (2.16) follows from the fact that there is $c_{3}>1$ big enough such that

$$
\frac{a_{k}}{a_{k-1}} \leq \frac{b_{k}}{b_{k-1}} \leq \frac{c_{0} b_{k}}{b_{k}-a_{k}}=\frac{c_{0}}{1-\left(a_{k} / b_{k}\right)} \leq c_{3}
$$

by (iii). It follows from (2.16) that there exists a constant $K>1$ such that

$$
\log b_{m} \leq c_{2} \sum_{k=k_{0}}^{m} \log \left(c_{0}+\frac{c_{3} a_{k}}{b_{k-1}}\right) \leq K \sum_{k=k_{0}}^{m} \frac{c_{3}^{2} a_{k}}{b_{k}}
$$

Therefore, we have

$$
\sum_{k=1}^{m} P\left(\mathbb{B}_{k}\right) \geq \frac{\varepsilon}{K c_{3}^{2}}\left(\log b_{m}\right)^{\varepsilon} \rightarrow \infty \quad \text { as } m \rightarrow \infty
$$

that is,

$$
\sum_{k=1}^{\infty} P\left(\mathbb{B}_{k}\right)=\infty
$$

This implies $\sum_{k=1}^{\infty} P\left(\mathbb{A}_{k}\right)=\infty$ and hence the condition (a) of Lemma 2.1 is satisfied.

Next, let us prove that condition (b) of Lemma 2.1 holds when $\left\{X_{k}\right\}$ is an LNQD random sequence. By the definition of $\left\{n_{k}\right\}_{k=1}^{\infty}$, two sets $A:=$ $\left\{j^{\prime} ; a_{j}+1 \leq j^{\prime} \leq a_{j}+b_{j}\right\}$ and $B:=\left\{k^{\prime} ; b_{k}+1 \leq k^{\prime} \leq 2 b_{k}\right\}$ for $j<k$, are disjoint. If we put

$$
\lambda_{j}=\frac{1}{\sigma\left(b_{j}\right) \beta\left(n_{j}\right)} \quad \text { and } \quad \lambda_{k}=\frac{1}{\sigma\left(b_{k}\right) \beta\left(n_{k}\right)},
$$

then it follows from the stationarity of $\left\{X_{k}\right\}$ that

$$
\begin{aligned}
& P\left(\mathbb{A}_{j} \cap \mathbb{A}_{k}\right) \\
& =P\left\{\frac{X_{a_{j}+1}+\cdots+X_{a_{j}+b_{j}}}{\sigma\left(b_{j}\right) \beta\left(n_{j}\right)}>\sqrt{1-4 \varepsilon}, \frac{X_{b_{k}+1}+\cdots+X_{2 b_{k}}}{\sigma\left(b_{k}\right) \beta\left(n_{k}\right)}>\sqrt{1-4 \varepsilon}\right\} \\
& =P\left\{\sum_{j^{\prime} \in A} \lambda_{j} X_{j^{\prime}}>\sqrt{1-4 \varepsilon}, \sum_{k^{\prime} \in B} \lambda_{k} X_{k^{\prime}}>\sqrt{1-4 \varepsilon}\right\} \\
& \leq P\left\{\sum_{j^{\prime} \in A} \lambda_{j} X_{j^{\prime}}>\sqrt{1-4 \varepsilon}\right\} P\left\{\sum_{k^{\prime} \in B} \lambda_{k} X_{k^{\prime}}>\sqrt{1-4 \varepsilon}\right\} \\
& =P\left\{\frac{X_{a_{j}+1}+\cdots+X_{a_{j}+b_{j}}}{\sigma\left(b_{j}\right) \beta\left(n_{j}\right)}>\sqrt{1-4 \varepsilon}\right\} P\left\{\frac{X_{b_{k}+1}+\cdots+X_{2 b_{k}}}{\sigma\left(b_{k}\right) \beta\left(n_{k}\right)}>\sqrt{1-4 \varepsilon}\right\} \\
& =P\left\{\frac{X_{1}+\cdots+X_{b_{j}}}{\sigma\left(b_{j}\right) \beta\left(n_{j}\right)}>\sqrt{1-4 \varepsilon}\right\} P\left\{\frac{X_{1}+\cdots+X_{b_{k}}}{\sigma\left(b_{k}\right) \beta\left(n_{k}\right)}>\sqrt{1-4 \varepsilon}\right\} \\
& =P\left(\mathbb{A}_{j}\right) P\left(\mathbb{A}_{k}\right) .
\end{aligned}
$$

This inequality yields that condition (b) of Lemma 2.1 is satisfied and hence (2.15) holds true by Lemma 2.1. 
On the other hand, assume that $\left\{X_{k} ; k=1,2, \cdots\right\}$ is a centered strictly stationary LPQD random sequence with conditions (i)-(ii). Set

$$
\mathbb{A}_{k}^{\prime}=\left\{U_{k}>\sqrt{1-3 \varepsilon} \beta\left(n_{k}\right)\right\}
$$

We will show that

$$
P\left(\mathbb{A}_{k}^{\prime} \text {, i.o. }\right)=1 \text {. }
$$

Choose a differential function $f(x)$ on $\mathbb{R}$ such that $\left|f^{\prime}(x)\right| \leq \kappa$ for some $0<$ $\kappa<\infty$ and

$$
0 \leq I\left\{x>\sqrt{1-2 \varepsilon} \beta\left(n_{k}\right)\right\} \leq f(x) \leq I\left\{x>\sqrt{1-3 \varepsilon} \beta\left(n_{k}\right)\right\} \leq 1 .
$$

In order to prove $(2.18)$, it is enough to show that

$$
\sum_{k=1}^{\infty} f\left(U_{k}\right)=\infty \quad \text { a.s. }
$$

From (2.17) and (2.19), we get

$$
\sum_{k=1}^{\infty} E f\left(U_{k}\right) \geq \sum_{k=1}^{\infty} P\left(\mathbb{B}_{k}\right)=\infty .
$$

By Markov's inequality, we have

$$
\begin{aligned}
& P\left\{\sum_{k=1}^{\infty} f\left(U_{k}\right)<\frac{1}{2} \sum_{k=1}^{n} E f\left(U_{k}\right)\right\} \\
& \quad \leq P\left\{\left|\sum_{k=1}^{n} f\left(U_{k}\right)-\sum_{k=1}^{n} E f\left(U_{k}\right)\right|>\frac{1}{2} \sum_{k=1}^{n} E f\left(U_{k}\right)\right\} \\
& \leq 4 \operatorname{Var}\left(\sum_{k=1}^{n} f\left(U_{k}\right)\right) /\left(\sum_{k=1}^{n} E f\left(U_{k}\right)\right)^{2} \\
& \leq \frac{4}{\sum_{k=1}^{n} E f\left(U_{k}\right)}+\frac{8 \sum_{k=1}^{\infty} \sum_{j=k+1}^{\infty}\left|\operatorname{Cov}\left(f\left(U_{k}\right), f\left(U_{j}\right)\right)\right|}{\left(\sum_{k=1}^{n} E f\left(U_{k}\right)\right)^{2}} .
\end{aligned}
$$

For $\theta>1$, let $b_{k}=\theta^{k}, k \geq 1$. Noting that $U_{k}$ and $U_{j}$ are LPQD from the definition of LPQD, it follows from $(\mathrm{i}),\left(P_{1}\right),\left(P_{2}\right)$ and the regularity of $\sigma(\cdot)$ 
that

$$
\begin{aligned}
& \sum_{k=1}^{\infty} \sum_{j=k+1}^{\infty}\left|\operatorname{Cov}\left(f\left(U_{k}\right), f\left(U_{j}\right)\right)\right| \\
& \leq \sum_{k=1}^{\infty} \sum_{j=k+1}^{\infty} \int_{-\infty}^{\infty} \int_{-\infty}^{\infty}\left|f^{\prime}(x)\right|\left|f^{\prime}(y)\right|\left(P\left\{U_{k} \geq x, U_{j} \geq y\right\}\right. \\
& \left.\quad-P\left\{U_{k} \geq x\right\} P\left\{U_{j} \geq y\right\}\right) d x d y \\
& \leq \kappa^{2} \sum_{k=1}^{\infty} \sum_{j=k+1}^{\infty} \operatorname{Cov}\left(U_{k}, U_{j}\right) \leq c \sum_{k=1}^{\infty} \frac{1}{\sigma^{2}\left(b_{k}\right)} \sum_{j=k+1}^{\infty} \operatorname{Cov}\left(S_{b_{k}}, S_{b_{j}}\right) \\
& \leq c \sum_{k=1}^{\infty} \frac{b_{k}}{\sigma^{2}\left(b_{k}\right)} \sum_{j=k+1}^{\infty} \operatorname{Cov}\left(X_{1}, S_{b_{j}}\right) \\
& \leq c \sum_{k=1}^{\infty} \theta^{k(1-2 \alpha)} b_{k+1} \sum_{b_{j} \geq b_{k}+1} \operatorname{Cov}\left(X_{1}, X_{b_{j}}\right) \\
& \leq c \sum_{k=1}^{\infty} \theta^{k(1-2 \alpha)} \theta^{k+1} b_{k}^{-\lambda} \leq c \sum_{k=1}^{\infty} \theta^{k(1-2 \alpha)} \theta^{k} \theta^{-\lambda k} \\
& =c \sum_{k=1}^{\infty} \theta^{-(\lambda-2+2 \alpha) k}<\infty
\end{aligned}
$$

for $\alpha>0$ and $\lambda>2$. Combining (2.21)-(2.23) and letting $n \rightarrow \infty$ yields

$$
P\left\{\sum_{k=1}^{\infty} f\left(U_{k}\right)<\infty\right\}=0 .
$$

This proves (2.20) and consequently (2.18). Let

$$
\mathbb{A}_{k}^{\prime \prime}=\left\{\frac{S_{b_{k / 2}}}{\sigma\left(b_{k / 2}\right)} \geq-2 \beta\left(n_{k / 2}\right)\right\} .
$$

It follows from (1.7) and (2.18) that $P\left(\mathbb{A}_{k}^{\prime} \cap \mathbb{A}_{k}^{\prime \prime}\right.$, i.o. $)=1$. By the stationarity of $\left\{X_{k}\right\}$, it is obvious that

$$
\begin{aligned}
& P\left\{\frac{U_{k}}{\beta\left(n_{k}\right)}>\sqrt{1-4 \varepsilon}, \text { i.o. }\right\}=P\left\{\frac{S_{b_{k}}}{\sigma\left(b_{k}\right)}>\sqrt{1-4 \varepsilon} \beta\left(n_{k}\right), \text { i.o. }\right\} \\
& \quad \geq P\left\{\frac{S_{b_{k}}}{\sigma\left(b_{k}\right)}>\sqrt{1-3 \varepsilon} \beta\left(n_{k}\right)-2 \beta\left(n_{k / 2}\right), \text { i.o. }\right\} \geq P\left\{\mathbb{A}_{k}^{\prime} \cap \mathbb{A}_{k}^{\prime \prime}, \text { i.o. }\right\}=1
\end{aligned}
$$

for $k$ large enough. This implies (2.15) and completes the proof of Theorem 1.2 . 


\section{Appendix: Proof of Proposition 2.3}

First we prove an auxiliary result for strictly stationary random field to get Proposition 2.3 .

Lemma 3.1. Let $\mathbb{D}$ be a compact subset of $\mathbb{R}_{+}^{d}$ and let $\left\{X_{\mathbf{t}} ; \mathbf{t}:=\left(t_{1}, \cdots, t_{d}\right) \in\right.$ $\mathbb{D}\}$ be a separable and centered strictly stationary random field with $d$-dimensional indices. For any $\mathbf{u}<\mathbf{v}$ in $\mathbb{D}$, denote by $X(\mathbf{u}, \mathbf{v}]$ a random field generated by $\left\{X_{\mathbf{t}} ; \mathbf{t} \in \mathbb{D}\right\}$ on $d$-cube $(\mathbf{u}, \mathbf{v}] \subset \mathbb{D}$ such that

$$
0<\Gamma:=\sup _{\mathbf{u}, \mathbf{v} \in \mathbb{D}} \sqrt{E\{X(\mathbf{u}, \mathbf{v}]\}^{2}}<\infty \text { and } \sqrt{E\{X(\mathbf{u}, \mathbf{v}]\}^{2}} \leq \varphi(\mid \mathbf{v}-\mathbf{u} \|) \text {, }
$$

where we denote $\|\mathbf{p}\|=p_{1} \cdots p_{d}$ for $\mathbf{p}:=\left(p_{1}, \cdots, p_{d}\right) \in \mathbb{R}_{+}^{d}$ and $\varphi(h)$ is a nondecreasing continuous function of $h>0$. Then, for any $\lambda>0$ and $K_{1}>$ $(2 \sqrt{2}+2) \sqrt{1+d \log 3}$, there exists a constant $c>0$ such that

$$
\begin{aligned}
& P\left\{\sup _{\mathbf{u}, \mathbf{v} \in \mathbb{D}}|X(\mathbf{u}, \mathbf{v}]| \geq x\left(\Gamma+K_{1} \int_{0}^{\infty} \varphi\left(\lambda^{d / 2} 2^{-d y^{2} / 2}\right) d y\right)\right\} \\
& \leq c \frac{m(\mathbb{D})}{\lambda^{d}}\left(P\left\{\frac{|X(\mathbf{0}, \mathbf{v}]|}{\sqrt{E\{X(\mathbf{0}, \mathbf{v}]\}^{2}}} \geq x\right\}\right. \\
& \left.\quad+\sum_{n=1}^{\infty} 2^{d 2^{n}} P\left\{\frac{|X(\mathbf{0}, \mathbf{v}]|}{\sqrt{E\{X(\mathbf{0}, \mathbf{v}]\}^{2}}} \geq x \sqrt{1+d \log 3} \cdot 2^{n / 2}\right\}\right)
\end{aligned}
$$

for $x \geq 1$, where $m(\mathbb{D})$ denotes the Lebesgue measure of $\mathbb{D}$.

Proof. For each $n=0,1,2, \cdots$, put $\varepsilon_{n}=\lambda 2^{-2^{n}}, \lambda>0$. Denote a diameter of any subset $A$ of $\mathbb{D}$ by $\rho(A)$. Let $\left\{T_{i}^{(n)} ; i=1,2, \cdots, N_{\varepsilon_{n}}(\mathbb{D})\right\}$ be a minimal $\varepsilon_{n^{-}}$ net of $\mathbb{D}$, where $N_{\varepsilon_{n}}(\mathbb{D})=\min \left\{k ; \mathbb{D} \subset \cup_{i=1}^{k} T_{i}^{(n)}, \rho\left(T_{i}^{(n)}\right) \leq \varepsilon_{n}\right\}$. Then there is a positive constant $c$ such that $N_{\varepsilon_{n}}(\mathbb{D}) \leq c \frac{m(\mathbb{D})}{\varepsilon_{n}^{d}}$. Set $\Delta_{n}=\cup_{i=1}^{N_{\varepsilon_{n}}(\mathbb{D})}\left\{\mathbf{t}_{i}^{(n)}\right\}$ for all $\mathbf{t}_{i}^{(n)} \in T_{i}^{(n)}$. Let $K_{2}>\sqrt{1+d \log 3}$ and $K_{1}=(2 \sqrt{2}+2) K_{2}$. For $x \geq 1$, set

$$
x_{k}=x K_{2} \varphi\left(\varepsilon_{k-1}^{d / 2}\right) 2^{k / 2}, \quad k \geq 1 .
$$

Letting $\delta_{k}=2^{(k-1) / 2}$ for $k \geq 0$, it is clear that

$$
2^{k / 2}=(2 \sqrt{2}+2)\left(\delta_{k}-\delta_{k-1}\right) .
$$

Thus we have

$$
\begin{aligned}
\sum_{k=1}^{\infty} x_{k} & =x K_{1} \sum_{k=1}^{\infty} \varphi\left(\lambda^{d / 2} 2^{-d \delta_{k}^{2} / 2}\right)\left(\delta_{k}-\delta_{k-1}\right) \\
& \leq x K_{1} \sum_{k=1}^{\infty} \int_{\delta_{k-1}}^{\delta_{k}} \varphi\left(\lambda^{d / 2} 2^{-d y^{2} / 2}\right) d y \\
& \leq x K_{1} \int_{0}^{\infty} \varphi\left(\lambda^{d / 2} 2^{-d y^{2} / 2}\right) d y
\end{aligned}
$$


Therefore, we conclude

$$
\begin{aligned}
& P\left\{\sup _{\mathbf{u}, \mathbf{v} \in \mathbb{D}}|X(\mathbf{u}, \mathbf{v}]| \geq x\left(\Gamma+K_{1} \int_{0}^{\infty} \varphi\left(\lambda^{d / 2} 2^{-d y^{2} / 2}\right) d y\right)\right\} \\
& \leq P\left\{\sup _{\mathbf{u}, \mathbf{v} \in \mathbb{D}}|X(\mathbf{u}, \mathbf{v}]| \geq x \Gamma+\sum_{k=1}^{\infty} x_{k}\right\} \\
& \quad \leq \lim _{n \rightarrow \infty} P\left\{\sup _{\mathbf{u}, \mathbf{v} \in \Delta_{n}}|X(\mathbf{u}, \mathbf{v}]| \geq x \Gamma+\sum_{k=1}^{n} x_{k}\right\} .
\end{aligned}
$$

Let

$$
\begin{aligned}
& B_{0}=\left\{\sup _{\mathbf{u}, \mathbf{v} \in \Delta_{0}}|X(\mathbf{u}, \mathbf{v}]| \geq x \Gamma\right\} \text { and } \\
& B_{n}=\left\{\sup _{\mathbf{u}, \mathbf{v} \in \Delta_{n}}|X(\mathbf{u}, \mathbf{v}]| \geq x \Gamma+\sum_{k=1}^{n} x_{k}\right\}, \quad n \geq 1 .
\end{aligned}
$$

By induction, we have

$$
P\left(B_{n}\right)=P\left(B_{n} \cap B_{n-1}\right)+P\left(B_{n} \cap B_{n-1}^{c}\right) \leq P\left(B_{0}\right)+\sum_{n=1}^{\infty} P\left(B_{n} \cap B_{n-1}^{c}\right) .
$$

By the way, for each $n \geq 1$, we have

$$
\begin{aligned}
& P\left(B_{n} \cap B_{n-1}^{c}\right) \\
& \leq P\left\{\bigcup_{\mathbf{u}, \mathbf{v} \in \Delta_{n}}\left\{2|X(\mathbf{u}, \mathbf{v}]| \geq x \Gamma+\sum_{k=1}^{n} x_{k}\right\}\right. \\
& \left.\quad \bigcap_{\mathbf{u}, \mathbf{v} \in \Delta_{n-1}}\left\{|X(\mathbf{u}, \mathbf{v}]|<x \Gamma+\sum_{k=1}^{n-1} x_{k}\right\}\right\} \\
& \left.\quad \leq \sum_{\mathbf{v} \in \Delta_{n} \quad \mathbf{u} \in \Delta_{n-1}-1} \sum_{|\mathbf{v}-\mathbf{u}| \leq \varepsilon_{n-1}^{d / 2}} P\left\{|X(\mathbf{u}, \mathbf{v}]| \geq x_{n}\right\}\right\} \\
& \quad \leq c \frac{\bigcup_{\mathbf{u} \in \Delta_{n-1}}(\mathbb{D})}{\varepsilon_{n}^{d}} P\left\{\frac{|X(\mathbf{u}, \mathbf{v}]|}{\sqrt{E\left\{X(\mathbf{u}, \mathbf{v}] \varepsilon_{n-1}^{d}\right.}} \geq \frac{x_{n}}{\varphi(\|\mathbf{v}-\mathbf{u}\|)}\right\} \\
& \leq c \frac{m(\mathbb{D})}{\varepsilon_{n}^{d}} P\left\{\frac{|X(\mathbf{u}, \mathbf{v}]|}{\sqrt{E\{X(\mathbf{u}, \mathbf{v}]\}^{2}}} \geq x K_{2} 2^{n / 2}\right\}
\end{aligned}
$$




$$
\begin{aligned}
& \leq c \frac{m(\mathbb{D})}{\lambda^{d}} 2^{d 2^{n}} P\left\{\frac{|X(\mathbf{u}, \mathbf{v}]|}{\sqrt{E\{X(\mathbf{u}, \mathbf{v}]\}^{2}}} \geq x \sqrt{1+d \log 3} \cdot 2^{n / 2}\right\} \\
& \leq c \frac{m(\mathbb{D})}{\lambda^{d}} 2^{d 2^{n}} P\left\{\frac{|X(\mathbf{0}, \mathbf{v}]|}{\sqrt{E\{X(\mathbf{0}, \mathbf{v}]\}^{2}}} \geq x \sqrt{1+d \log 3} \cdot 2^{n / 2}\right\}
\end{aligned}
$$

and

$$
\begin{aligned}
& \sum_{n=1}^{\infty} P\left(B_{n} \cap B_{n-1}^{c}\right) \\
& \quad \leq c \frac{m(\mathbb{D})}{\lambda^{d}} \sum_{n=1}^{\infty} 2^{d 2^{n}} P\left\{\frac{|X(\mathbf{0}, \mathbf{v}]|}{\sqrt{E\{X(\mathbf{0}, \mathbf{v}]\}^{2}}} \geq x \sqrt{1+d \log 3} \cdot 2^{n / 2}\right\},
\end{aligned}
$$

where $c>0$ is a constant. On the other hand, we have

$$
\begin{aligned}
P\left(B_{0}\right) & \leq c \frac{m(\mathbb{D})}{\varepsilon_{0}^{d}} P\{|X(\mathbf{u}, \mathbf{v}]| \geq x \Gamma\} \\
& \leq c \frac{m(\mathbb{D})}{\lambda^{d}} P\left\{\frac{|X(\mathbf{u}, \mathbf{v}]|}{\sqrt{E\{X(\mathbf{u}, \mathbf{v}]\}^{2}}} \geq x\right\} \leq c \frac{m(\mathbb{D})}{\lambda^{d}} P\left\{\frac{|X(\mathbf{0}, \mathbf{v}]|}{\sqrt{E\{X(\mathbf{0}, \mathbf{v}]\}^{2}}} \geq x\right\} .
\end{aligned}
$$

This completes the proof of Lemma 3.1.

Proof of Proposition 2.3. For given $b_{n}(n \geq 1)$, we set $\mathbb{D}_{n}=\{(i, j) \in$ $\left.\mathbb{Z}_{+}^{2} ; 0 \leq i \leq b_{n} 1 \leq j \leq b_{n}\right\}$. To apply Lemma 3.1, put

$$
X(i, i+j]=\frac{S_{i+j}-S_{i}}{\sigma\left(b_{n}\right)} \text { and } \varphi(z)=\frac{\sigma(z)}{\sigma\left(b_{n}\right)} \text { for } z>0,
$$

where $\sigma\left(b_{n}\right):=\sqrt{E\left\{S_{b_{n}}\right\}^{2}}$. It is clear that

$$
\begin{gathered}
E\{X(i, i+j]\}=0, \quad \sqrt{E\{X(i, i+j]\}^{2}}=\frac{\sigma(j)}{\sigma\left(b_{n}\right)}=\varphi(j) \\
\text { and } \Gamma=\sup _{(i, j) \in \mathbb{D}_{n}} \sqrt{E\{X(i, i+j]\}^{2}}=1 .
\end{gathered}
$$

Since $\sigma(\cdot)$ is a nondecreasing continuous and regularly varying function with exponent $\alpha>0$ at $\infty$, it follows that, for any $\varepsilon>0$, there exists a constant $C_{\varepsilon}>0$ such that

$$
\begin{aligned}
K_{1} \int_{0}^{\infty} \varphi\left(C_{\varepsilon} b_{n} 2^{-y^{2}}\right) d y & =K_{1} \int_{0}^{\infty} \frac{\sigma\left(C_{\varepsilon} b_{n} 2^{-y^{2}}\right)}{\sigma\left(b_{n}\right)} d y \\
& \leq K_{1} \int_{0}^{\infty}\left(C_{\varepsilon} 2^{-y^{2}}\right)^{\alpha} d y<\varepsilon
\end{aligned}
$$


where $K_{1}>(2 \sqrt{2}+2) \sqrt{1+2 \log 3}$ and we take $\lambda=C_{\varepsilon} b_{n}$ in Lemma 3.1. Set $u=x(1+\varepsilon)$ for $x \geq 1$. Then, by (3.1) and the stationarity of $\left\{X_{k}\right\}$, we have

$$
\begin{gathered}
P\left\{\sup _{0 \leq i \leq b_{n}} \sup _{1 \leq j \leq b_{n}} \frac{\left|S_{i+j}-S_{i}\right|}{\sigma\left(b_{n}\right)} \geq u\right\}=P\left\{\sup _{(i, j) \in \mathbb{D}_{n}}|X(i, i+j]| \geq u\right\} \\
\leq P\left\{\sup _{(i, j) \in \mathbb{D}_{n}}|X(i, i+j]| \geq x\left(1+K_{1} \int_{0}^{\infty} \varphi\left(C_{\varepsilon} b_{n} 2^{-y^{2}}\right) d y\right)\right\} \\
\leq c \frac{b_{n}^{2}}{\left(C_{\varepsilon} b_{n}\right)^{2}}\left(P\left\{\frac{\left|X\left(0, b_{n}\right]\right|}{\sqrt{E\left\{X\left(0, b_{n}\right]\right\}^{2}}} \geq x\right\}\right. \\
\left.\quad+\sum_{n=1}^{\infty} 2^{2^{n+1}} P\left\{\frac{\left|X\left(0, b_{n}\right]\right|}{\sqrt{E\left\{X\left(0, b_{n}\right]\right\}^{2}}} \geq x \sqrt{1+2 \log 3} \cdot 2^{n / 2}\right\}\right) \\
\leq c_{\varepsilon}\left(P\left\{\frac{\left|S_{b_{n}}\right|}{\sigma\left(b_{n}\right)} \geq \frac{u}{1+\varepsilon}\right\}\right. \\
\left.\quad+\sum_{n=1}^{\infty} 2^{2^{n+1}} P\left\{\frac{\left|S_{b_{n}}\right|}{\sigma\left(b_{n}\right)} \geq \frac{u}{1+\varepsilon} \sqrt{1+2 \log 3} \cdot 2^{n / 2}\right\}\right),
\end{gathered}
$$

where $c_{\varepsilon}$ is a positive constant depending only on $\varepsilon>0$. This completes the proof.

\section{References}

[1] T. Birkel, A Functional Central Limit Theorem for Positively Dependent Random Variables, J. Multivariate Anal. 44 (1993), no. 2, 314-320.

[2] Y. K. Choi and M. Csörgő, Path properties of $l^{p}$-valued Gaussian random fields, Sci. China Ser. A: Math. 50 (2007), 1501-1520.

[3] _ Limsup results and LIL for partial sum processes of a Gaussian random field, Acta Math. Sinica. 24 (2008), no. 9, 1497-1506.

[4] Y. K. Choi, K. S. Hwang, T. S. Kim, Z. Y. Lin and W. S. Wang, Asymptotic behaviors for partial sum processes of a Gaussian sequence, Acta Math. Hungar. 103 (2004), $43-54$.

[5] M. Csörgö, Z. Y. Lin, and Q. M. Shao, Path properties for $l^{\infty}$-valued Gaussian processes, Proc. Amer. Math. Soc. 121 (1994), 225-236.

[6] M. Csörgö and P. Révész, Strong Approximations in Probability and Statistics, Academic Press, New York, 1981.

[7] _ How big are the increments of a Wiener process? Ann. Probab. 7 (1979), 731-737.

[8] _ How small are the increments of a Wiener process? Stochastic Process Appl. 8 (1979), 119-129.

[9] M. Csörgő and J. Steinebach, Improved Erdös-Rényi and strong approximation laws for increments of partial sums, Ann. Probab. 9 (1981), no. 6, 988-996.

[10] P. Deheuvels and J. Steinebach, Exact convergence rates in strong approximation laws for large increments of partial sums, Probab. Theory Related Fields 76 (1987), 369-393.

[11] P. Erdös and A. Rényi, On a new law of large numbers, J. Analyse Math. 13 (1970), $103-111$.

[12] J. Esary, F. Proschan and D. Walkup, Association of random variables with applications, Ann. Math. Statist. 38 (1967), no. 4, 1466-1474. 
[13] K. Joag-Dev, Independence via uncorrelatedness under certain dependence structures, Ann. Probab. 11 (1983), no. 4, 1037-1041.

[14] K. Joag-Dev and F. Proschan, Negative association of random variables with applications, Ann. Statist. 11 (1983), no. 1, 286-295.

[15] H. Lanzinger and U. Stadtmüller, Maxima of increments of partial sums for certain subexponential distributions, Stochastic Process. Appl. 86 (2000), 307-322.

[16] E. L. Lehmann, Some concepts od dependence, Ann. Math. Statist. 37 (1966), no. 5, $1137-1153$.

[17] Y. X. Li and J. F. Wang, The law of the iterated logarithm for positively dependent random variables, J. Math. Anal. Appl. 339 (2008), no. 1, 259-265.

[18] Z. Y. Lin, On Csörgö-Révész's increments of sums of non-i.i.d. random variables, Scientia Sinica (Series A) 30 (1987), 921-931.

[19] On the increments of partial sums of $\phi$-mixing sequence, Teor. Veroyatnost. i Primenen. 36 (1991), 326-336; translation in Theory Probab. Appl. 36 (1992), 316-328.

[20] Z. Y. Lin, S. H. Lee, K. S. Hwang and Y. K. Choi, Some limit theorems on the increments of $l^{p}$-valued multi-parameter Gaussian processes, Acta Math. Sinica, English Ser. 20 (2004), no. 6, 1019-1028.

[21] Z. Y. Lin and C. R. Lu, Strong Limit Theorems, New York, 1975.

[22] Z. Y. Lin, C. R. Lu and L. X. Zhang, Path Properties of Gaussian Processes, Zhejiang University Press, 2001.

[23] C. M. Newman, Normal fluctuations and the FKG inequalities, Comm. Math. Phys. 74 (1980), no. 2, 119-128.

[24] _ Asymptotic independence and limit theorems for positively and negatively dependent random variables, in: Inequalities in Statistics and Probability (Tong Y. L., Ed., Institute of Mathematical Statistics, Hayward, CA) (1984), 127-140.

[25] V. V. Petrov, Sums of Independent Random Variables, Springer-Verlag, New York, 1975.

[26] J. F. Wang and L. X. Zhang, A Berry-Esseen theorem for weakly negatively dependent random variables and its applications, Acta Math. Hungar. 110 (2006), no, 4, 293-308.

[27] Y. Yang and Y. B. Wang, The asymptotical normality of the renewal process generated by strictly stationary LPQD sequences, Chinese J. Appl. Probab. Statist. 24 (2008), no. $1,37-42$.

[28] L. X. Zhang, Central limit theorems for asymptotically negatively associated random fields, Acta Math. Sinica, Engl. Ser. 16 (2000), no. 4, 691-710.

First Author

Department of Mathematics and Rins, Gyeongsang National University, Jinju 660-701, SOUth Korea

E-mail address: hankm21han@daum.net

Second Author

Department of Mathematics and RINS, Gyeongsang National University, Jinju 660-701, SOUth Korea

E-mail address: mhj0307@hanmail.net

CORRESPONDING AUTHOR

Department of Mathematics and Rins, Gyeongsang National University, Jinju 660-701, SOUth Korea

E-mail address: mathykc@naver.com 